\title{
JÓVENES MIGRANTES Y POLITICA: LOS NUEVOS ROSTROS DE LA BOLIVIANEIDAD EN ARGENTINA ${ }^{1}$
}

\author{
Alfonso Hinojosa GORDONAVA²
}

RESUMEN: El artículo trata sobre las nuevas organizaciones de jóvenes migrantes bolivianos en el presente siglo y cómo responden a nuevas lógicas organizativas que van más allá del anclaje nacionalista de los y las trabajadores migrantes. Este escenario es resultado de procesos de politización que responden tanto a dinámicas en los países de origen, pero cada vez más a los contextos sociales, económicos y políticos en los lugares de destino. Asimismo, la emergencia de nuevos horizontes y actores de lucha migrante ligado sobre todo a identidades laborales sobrepasan los guetos nacionalistas, pero también al potencial femenino de reivindicaciones de género en articulación con lo migrante.

PALABRAS CLAVE: jóvenes migrantes; bolivianos en Argentina; política y migración; sujeto político migrante.

\section{YOUNG MIGRANTS AND POLITICS: THE NEW EXPRESSIONS OF 'BOLIVIANESS' IN ARGENTINA}

ABSTRACT: The article deals with new organizations of young Bolivian migrants in Buenos Aires in the present century and how they respond to new organizational logics that go beyond the nationalist anchoring of migrant workers, being the result of politicization processes that respond both to dynamics in the countries of origin, but increasingly to the social, economic and political contexts in the places of destination. Likewise, it shows the

\footnotetext{
1 El presente artículo se lo realizó en el marco de una investigación financiada por el Instituto de Investigación e Interacción Social y Postgrado (IIISP) de la Carrera de Trabajo Social de la UMSA.

2 Universidad Mayor de San Andrés. La Paz - Bolivia. Docente e investigador. alf_hg@yahoo.com.
} 
emergence of new horizons and actors of migrant demand based on labor identities that go beyond nationalist ghettos, as well as the organizational potential of women with gender demands and in bondingwith migration.

KEYWORDS: young migrants; bolivians in Argentina; politics and migration; migrant political subject.

\section{Los datos en el presente siglo}

El inicio del presente siglo ha significado para Bolivia profundas transformaciones en diversos ordenes de la sociedad. De manera específica las dinámicas poblacionales y geográficas han hecho del espacio urbano el mayor concentrador de habitantes en base a un intenso proceso de migración interna, que a su vez ha estado ligada a la emigración internacional. En los últimos cuarenta años no sólo se incrementaron las migraciones internas en el país consolidando un eje central, compuesto por las ciudades de La Paz, Cochabamba y Santa Cruz, sino que también - más recientemente - se han fortalecido todo un sistema de ciudades intermedias que consolidan las tendencias de urbanización, donde éstas se hallan geográficamente más cercanas a los espacios rurales, pero económica y cultural fuertemente ligadas a la ciudad. De manera paralela, se ha incrementado la emigración al exterior a la vez que también se han diversificado, ampliado y consolidado nichos laborales en países fronterizos, como en otros más allá de nuestras fronteras (sobre todo en España), haciendo de la emigración internacional una estrategia que se actualiza constantemente adaptándose con gran versatilidad a los cambios.

Los desplazamientos poblacionales hacia el exterior, según las cifras que proporcionó el Censo de Población y Vivienda realizado el año 2012 y donde por primera vez se incorporó la variable de 'emigración internacional', a través de una pregunta que indagaba sobre si en los hogares, durante los últimos diez años, algunos miembros habrían emigrado al exterior. Si la respuesta era positiva, se abrían otras preguntas respecto al país de residencia de esa persona, su edad y sexo. En todo caso, estos resultados arrojados por el Censo dan un total de 487.995 bolivianos que, entre el 2002 al 2012, habría emigrado del país, prevaleciendo ligeramente las mujeres, con un 51.03 por ciento, frente a los varones, con un 48.97 por ciento. El destino principal de los emigrantes bolivianos en este período fue la República Argentina, con un 38,22 por ciento, seguido de Espańa, con un 23,88 por ciento; Brasil, con 13,18 por ciento; Chile, con 5,95 
por ciento, y Estados Unidos, con 4,20 por ciento. El restante veinte por ciento se dispersa en un abanico muy amplio de países (INE, 2021). Los departamentos que registran el mayor número de emigrantes son La Paz (27,5 por ciento), Cochabamba (23,2 por ciento) y Santa Cruz (22,7 por ciento). Este primer dato muestra como la región paceña de ser espacio privilegiado de recepción de migración interna pasa a la vez a ser origen de la principal emigración nacional. Corriente parecida, pero en menor cantidad, parece evidenciarse en el oriente boliviano, de donde también se dan emigraciones hacia el exterior.

Es importante subrayar que el mayor porcentaje de hogares con emigrantes se registra en viviendas de área urbana: 11,8 por ciento declara tener alguna persona que se encuentra en un país extranjero. Este dato nos muestra otro cambio fundamental de las últimas décadas y que tiene que ver con que la mayor parte de los y las emigrantes internacionales salen hoy en día de espacios urbanos o periurbanos y ya no exclusivamente de las áreas rurales. Es interesante analizar la emigración de los espacios periurbanos, ya que los mismos son el resultado de las dinámicas de migración interna hacia las ciudades y donde, en algunos casos según la antigüedad de la trayectoria migratoria, prevalecen todavía como prácticas y hábitos de muchos elementos de la ruralidad, de lo comunal, que luego terminan expresándose en espacios transnacionales. La edad de la población señala que el 64,5 por ciento de las personas que salieron del país tienen entre 15 a 29 ańos, ratificando que se trata de sectores jóvenes cuya mano de obra es aprovechada más allá de nuestras fronteras. Dentro este grupo, destacan los más jóvenes de 20 a 24 años, tanto hombres como mujeres, que alcanzan a 27 por ciento.

De manera específica, la movilidad poblacional boliviana en busca de trabajo en Argentina es histórica, remontándose incluso al periodo colonial, sin embargo, en la década de los años ochenta del siglo pasado. El flujo de migrantes bolivianos aumentó principalmente por la crisis económica que atravesaba Bolivia y por la implementación del programa de ajuste estructural neoliberal llevado adelante desde 1985. Los años noventa marcan un periodo de estabilidad y, en cierto modo, de auge migratorio en virtud de las características económicas de dolarización que asumió Argentina y, también, por la amnistía declarada en ese país (1999) que posibilitó la legalización de unos 110 mil bolivianos (la mayoría asentados en Buenos Aires). Durante el periodo mencionado, se consolidan y se ramifican muchas de las trayectorias migratorias anteriores que correspondían al tipo urbano-urbano, tanto así que fueron estas sólidas redes estructuradas las que amortiguaron los efectos de la crisis que vivió Argentina a finales del 2001. El tiempo transcurrido, tras la severa crisis argentina, estabiliza 
los flujos poblacionales entre ambos países, aunque está claro que los niveles de ahorro y remesas característicos del periodo de dolarización se han diluido (BENENCIA, 2007, 2008). Sin embargo, hoy en día, los nichos laborales que tienen que ver la producción hortícola y con el trabajo en la manufactura textil prácticamente están en manos de migrantes bolivianos, este último rubro es el que más se ha desarrollado en las últimas décadas y concentra mayor cantidad de mano de obra joven proveniente sobre todo de la región altiplánica de La Paz, que termina en los talleres textiles de Buenos Aires, como veremos más adelante.

Es importante subrayar algunos elementos que son fundamentales para el análisis de las interacciones entre los migrantes y los lugares de origen - debemos resaltar los procesos de "territorialización transnacional" entre los dos países y cuyos horizontes culturales e identitarios definen un perfil propio que involucran a más de una generación. También es digno de destacar los niveles organizativos que despliegan los bolivianos en sus diversas actividades y que son elementos que se desarrollan más adelante.

\section{La 'guerra del gas' y el voto en el exterior}

Como ya mencionamos, los acontecimientos políticos por los cuales atravesó Bolivia a principios del presente siglo fueron muy profundos e impactaron no sólo en la población del país, sino también en sus emigrantes. El año 2000 dio pie a la denominada 'guerra del agua', en Cochabamba, en contra de la privatización del agua potable y que marcó el inicio de una serie de protestas de diversos movimientos sociales que derivarían, años más tarde, en los sucesos de octubre de 2003, en la ciudad de El Alto, conocidos como la 'guerra del gas'. Fueron acontecimientos estructurantes en la historia reciente de Bolivia, derivando en la presidencia democrática de Evo Morales el año 2006 y manteniéndose durante casi 14 años, periodo que asumió el apelativo de "proceso de cambio".

Durante el desarrollo de toda esta serie de acontecimientos de orden político centrados en la ciudad de El Alto, pero irradiados en todo el territorio nacional y más allá, paralelamente y de manera silenciosa se producían también dinámicas emigratorias muy importantes hacia el exterior, como hemos podido evidenciar anteriormente. Esto señala cuan estructural es la migración para amplios sectores de la población de Bolivia y del eje metropolitano de la ciudad de La Paz, en particular, como respuesta inmediata a las crisis se acentúan las dinámicas poblaciones, en nuestro caso, hacia el exterior.

El conjunto de estos acontecimientos profundos y estructurantes de la Bolivia actual que se vivieron a inicios de siglo tuvo también impacto y reper- 
cusiones en los residentes bolivianos en el exterior. Con seguridad, podemos afirmar que los impactos y resignificaciones que surgieron de los acontecimientos de "la guerra del gas" de octubre del 2003 traspasaron las fronteras nacionales e irrumpieron con fuerza. Sobre todo en la colectividad boliviana en Argentina, multitudinarias manifestaciones se dieron en la ciudad de Buenos Aires, inaugurando así momentos y espacios transnacionales de participación política, en virtud de demandas ancladas estrictamente en lo nacional, es decir, la demanda central era nacionalización de los hidrocarburos. Gavazzo (2009) resalta que la presencia de migrantes provenientes de Bolivia en marchas masivas ha sido observada con curiosidad y sorpresa por algunos y con censura por otros. Por ejemplo, en 2003, a pocos días de la renuncia del entonces presidente de Bolivia, Gonzalo Sánchez de Losada, miles de bolivianos residentes en Buenos Aires se movilizaron desde el Congreso Nacional hasta la Embajada de Bolivia. Portaban una amplia gama de reclamos, desde muertos en enfrentamientos hasta las polémicas decisiones respecto de la explotación de los recursos naturales.

Pasada la "guerra del gas" y la caída del expresidente Sánchez de Lozada, las colectividades bolivianas movilizadas en la Argentina, que a estas alturas se hallaban fuertemente politizadas, se mantuvieron activas y expectantes al devenir de los hechos en el país. Participando de manera activa y creativa en momentos claves del acontecer boliviano, como fueron los Referéndums de consulta ciudadana, a partir de articular en varias ciudades y lugares de Argentina 'votaciones simbólicas', movilizaron a miles de compatriotas, además de alguna que otra movilización de apoyo al 'proceso de cambio'.

En este contexto surge la demanda del voto en el exterior con gran fuerza luego de las movilizaciones de octubre en Buenos Aires. Si bien la demanda específica no era nueva en sí, luego del 2003 se convierte en el gran aglutinador de todos esos procesos de politización vividos en virtud a los acontecimientos de Bolivia. Es así que el año 2004 es la Federación de Asociaciones Civiles de Bolivianos residentes en la Argentina que entablan y le gana un juicio al estado boliviano para que puedan acceder al derecho de voto en el exterior. Este hecho, derivado de los sucesos de la 'guerra del gas' de octubre del 2003 y de sus repercusiones en el exterior (sobre todo en la Argentina), marcaron a nuestro entender el inicio o la irrupción del hecho migratorio en la agenda política nacional. Ya en el nuevo Estado Plurinacional se evidencia, por un lado, el deseo de incluir en "lo político" a los que hasta hace poco se les negaba este derecho; y, por el otro, la estrategia funcional de sumar más apoyo al gobierno por la vía electoral. En todo caso, la continuidad del proyecto del voto desde el exterior está ligado a los procesos de documentación. 
La aprobación por el gobierno boliviano de una ley autorizando el voto en el exterior el año 2009 ilustra el cambio radical en la postura del Estado boliviano hacia su población en el exterior con la llegada al poder de Evo Morales. A pesar de los obstáculos contenidos en la ley para limitar el impacto del voto emigrante sobre los resultados de las elecciones presidenciales, la participación de más de 125.000 emigrantes en los comicios del 6 diciembre de dicho año marcó un explícito reconocimiento del papel que los emigrantes quieren seguir teniendo en Bolivia, a pesar de su ausencia en el territorio nacional. Este aspecto también se expresó en octubre del año 2014 en la segunda experiencia de votación en el exterior, donde más de 160 mil bolivianos/as emitieron su voto en 33 países (TSE). En este sentido, el voto en el exterior puede ser considerado como uno de los mayores éxitos de este nuevo periodo en lo concerniente al hecho migratorio internacional.

"El proceso de cambio en Bolivia es como que se abrió un camino hacia el interior de nosotros mismos" (ORLANDO, 2006).

[...] queremos apoyar el proceso de cambio de Evo Morales, estamos con él ... me hace sentir muy bien saber que estoy colaborando desde acá con mis paisanos, este presidente lo está mejorando, yo veo muchos cambios en Bolivia ... quiero volver a mi país ... [llanto]... (GREGORIA, 2006).

Lo que en cierta medida permite el surgimiento y desarrollo de nuevas formas de articulación de prácticas sociales y políticas en la colectividad boliviana en la Argentina es el denso tejido cultural que articula a la colectividad. Esta matriz cultural es el núcleo estructurante de las prácticas sociales en el exterior, pero también de los cambios y nuevos sentidos que se viven en función de las transformaciones en la sociedad de origen, es decir, en Bolivia.

Durante este intenso periodo que va del 2003 al 2009 van surgiendo nuevas organizaciones, sobre todo, de jóvenes migrantes cuya mirada y accionar rebasa lo estrictamente cultural folclórico y empiezan a debatir y activar posiciones políticas sobre identidades y pertenencias étnicas, el acontecer en Bolivia, pero también, y cada vez con mayor intensidad, su situación como migrantes en los lugares en los cuales viven y trabajan. El trabajo cada vez empieza a ser también un gran elemento de discusión al interior de estos grupos de migrantes que poco a poco van volcando sus miradas y esfuerzos organizativos hacia las condiciones y políticas locales en las cuales se desarrollan. 
Surge (el grupo Bolivia Marka) ante los acontecimientos que se daban en Bolivia en los últimos años pero de manera específica durante el año 2007 a partir los referendos departamentales sobre las autonomías. En una primera instancia es el Centro de Residentes Bolivianos quien busca aglutinar esfuerzos y acciones en reacción a lo que acontecía en Bolivia.... en dicha reunión asistió muy poca gente y pese ello hubo muchos desacuerdos (...) sin embargo, "ahí decidimos convocar a más gente, hacer marchas para que se sienta en Bolivia el apoyo de los de acá ... (ORLANDO, 2006).

Muchos de estos líderes migrantes jóvenes consideran que las organizaciones tradicionales de los bolivianos en Argentina y sus propios liderazgos se hallan agotados en su discurso y práctica exclusivamente culturalista y de sus pugnas internas altamente frecuentes; pero también reconocen la importancia de la experiencia que los líderes mayores han adquirido como base para orientar futuras iniciativas.

\section{La construcción del sujeto político migrante}

\section{Los talleres textiles}

Variados estudios detallan cómo los migrantes bolivianos asentados en Buenos Aires desarrollaron diversas estrategias, tanto para adquirir un trabajo, una vivienda y documentación, como para reunirse y construir en el nuevo contexto urbano lugares y prácticas de identificación, ya que en Buenos Aires hay múltiples ámbitos de producción y reconstrucción de identidades vinculados a la 'colectividad boliviana'. ${ }^{3}$ Es un tejido social diverso y disperso por distintas zonas de la ciudad que incluye bailantas, restaurantes, fiestas familiares y barriales, ligas de fútbol, programas de radio, asociaciones civiles, publicaciones, ferias y comercios de diferentes tipos, dando cuenta de múltiples espacios vinculados con la bolivianidad.

En el sector de la confección textil, nicho laboral migrante por excelencia, existe una tendencia global hacia la reorganización de la producción a través de diversas formas de subcontratación para que ésta sea cada vez más flexibilizada y descentralizada en gran medida a partir de la contratación de mujeres talleristas.

\footnotetext{
3 Roberto Benencia (1997, 2004, 2005, 2007, 2008), Roberto Benencia y Gabriela Karasik (1995), Alejandro Grimson (1999), Alejandro Grimson e Paz Soldán (2000), Martha Giorgis (2004), Susana Sassone et al. (2004), Sergio Caggiano (2001, 2005a, 2005b, 2008), Eduardo Domenech (2005), Eduardo Domenech e Magliano (2009), Natalia Gavazzo $(2016,2014)$.
} 
Las grandes empresas contratan a proveedores, quienes, a su vez, distribuyen trabajo a subcontratistas, muchos de los cuales operan en la economía denominada informal. La fuerte competitividad que vive el sector forzó un modelo de negocio más rápido, más flexible y más barato haciendo que la mayor sobre explotación recaiga en los y las trabajadoras finales.

Este modelo impone y traslada la presión sobre los talleres textiles y los proveedores, generando diversas consecuencias negativas, las cuales afectan seriamente las condiciones de trabajo, donde la producción se basa en la utilización de mano de obra intensiva. Este modelo produce, por consiguiente, graves consecuencias para los y las trabajadoras, que se manifiestan en el exceso de horas de trabajo. Además, provoca fatiga y stress, genera lesiones y abuso de horas extras sin previo aviso o no remuneradas, no se respetan los salarios mínimos, falta cobertura social (no se cubren pensiones ni bajas) y garantías de seguridad e higiene mínimas, posee subcontrataciones incontroladas y trabajo eventual o sin contrato.

En estos lugares se emplea a trabajadores que, por lo general, son inmigrantes bolivianos y bolivianas. Estos talleres son conocidos por las malas condiciones de trabajo que en ellos se implementan. Son unidades de producción donde se trabaja en condiciones de alta explotación; las familias contratadas para desarrollar la actividad viven en el mismo lugar donde trabajan, por lo que las jornadas laborales pueden extenderse hasta más de 16 horas diarias. La subcontratación y tercerización de las fases productivas con menor valor agregado facilitan la existencia de condiciones laborales informales en busca de un menor costo por prenda. La confección está muy atomizada, proliferan las pequeñas y medianas empresas y hay mayor competitividad.

En un primer momento, lo que produce la migración en los jóvenes que llegan a Buenos Aires es una suerte de vacío alrededor de uno mismo, uno de los primeros impactos que la migración produce es lo restringido que se ven los lazos sociales, se reducen los amigos, la familia. Pero en ese momento, en esa vulnerabilidad y en el nuevo espacio del 'taller', se les presentan las nuevas condiciones de vida: "a partir de ahora se va a trabajar así, se va a dormir acá, a partir de ahora se come así”. Todo eso se empieza a naturalizarse, porque es un proceso en todos los migrantes en ese momento de vulnerabilidad. El taller textil le soluciona tres cosas inmediatas al migrante: techo, comida y trabajo. Son esas tres cosas que un migrante necesita solucionar lo más rápido posible y el taller se lo soluciona en unas condiciones muy complicadas, es decir, en condiciones de explotación muy fuerte. 
En todo caso el mundo en torno al taller textil ha generado lecturas y aproximaciones controversiales que se expresan cuando se habla a partir de nociones como la de "trabajo esclavo", "en negro", "marginal" e "informal". Dichas interpretaciones tienden a soslayar la complejidad, los cambios e hibridaciones de los procesos, en los cuales intervienen y construyen de modo cotidiano los sectores migrantes involucrados, en procesos colectivos que por otra parte ya suman más de dos décadas de desarrollo en la actividad productiva laboral y económica local. Y es precisamente desde el ámbito de los talleres textiles de donde surge el Colectivo Simbiosis Cultural, como veremos ahora.

Juan Vásquez es un joven boliviano procedente del altiplano paceño que vive hace más de veinte años en Buenos Aires y, como él mismo relata, fueron sus padres le hicieron migrar a los nueve años durante la década de los años ochenta. El primer lugar donde llegó era un taller textil, a partir de eso se fueron dando los procesos de inserción en la escuela y en la secundaria, pero siempre teniendo bien en cuenta que una cosa era la casa que era al mismo tiempo el taller y otra cosa era lo que vivía fuera de la casa-taller. Juan reconoce, en su caso, el hecho de que insertarse en un colegio de Buenos Aires siendo niño le permitió mimetizarse para ser parte del grupo local. Sin embargo, aquellos los jóvenes que migran a más temprana edad, a partir de los 15 años, y que van directo a trabajar, tienen otra realidad que es la de armar un gueto entorno al taller: trabajar en el taller más de 12 horas diarias, escuchar lo que pasa en el taller, ir a los lugares donde están todos los que trabajan en talleres, etc. En todo caso, ahí se termina de armar un gueto que es muy fuerte y que además es lo que sostiene hoy por hoy el sistema de producción de la manufactura textil en Buenos Aires.

Por su parte, Delia Colque es otra joven boliviana nacida en la ciudad de El Alto que estando en la Universidad Mayor de San Andrés de La Paz, se vio obligada a migrar a Buenos Aires a principios del presente siglo a trabajar en el rubro textil. La decisión de emigrar se debió a situaciones económicas, pero sobre todo tratando de escapar de la violencia machista que reinaba en su familia, situación en la que se encuentran muchas familias. La migración de mujeres bolivianas hacia Argentina ha sido históricamente parte de un proyecto migratorio familiar, y si bien en los últimos años aumentó el número de mujeres que arribaron solas. Todavía, la mayor parte de este flujo se desplaza hacia este país en contextos familiares. Se establece también, en el caso de las manufacturas textiles de Buenos Aires, si bien se emplean en igual porcentaje hombres y mujeres, pareciera que en los últimos años habría un leve aumento de las mujeres.

Juan y Delia son fundadores y miembros activos del Colectivo Simbiosis Cultural. El "Colectivo Simbiosis Cultural" es una agrupación de jóvenes - boli- 
vianos en su gran mayoría - que hacia el año 2008 comienza a organizarse en el barrio de Flores, en la capital porteńa y está conformada sobre todo por costureros que cuestionan las formas de trabajo dentro de los talleres textiles en los cuales laburan junto con miles de otros bolivianos. En su block de internet se puede leer respecto a ellos y ellas:

Somos un colectivo que tiene sus raíces - de una forma u otra - en Bolivia, pero la extendió en Argentina

Somos ese híbrido de culturas,

Somos los chuequistas, somos las overlockas

Somos los retazos que se animaron a quedar fuera de ese maldito molde

No somos lo que nos dicen, ni lo que piensan, no nos vamos a hacer cargo de sus prejuicios, porque tenemos los nuestros

Somos un espacio en el que reconocemos a la bolivianeidad $\mathrm{La} /$ nos pensamos, la/nos cuestionamos y buscamos formas de dialogarla/nos A principios de ańo 2008 comenzamos con esta locura, comenzamos a soñar de una forma distinta a la que solíamos hacerlo, lo comenzamos a hacer de manera colectiva ${ }^{4}$.

La temática del trabajo textil siempre fue una preocupación central del Colectivo, comenzaron a discutirlo y hablarlo en sus diversidades y complejidades que van desde la vivienda-taller, las articulaciones entre medios de producción y las cadenas productivas, la mano de obra migrante, el folclorismo, los medios de comunicación de la colectividad, diversidades sexuales, género y violencia, etc. En estas dinámicas un elemento fue fundamental, abandonar la mirada y la postura victimista. Un hecho marcó sus vidas y la de sus compas. El año 2006 un incendio en la calle Luis Viale de Buenos Aires acabó con un taller textil y con la vida de seis personas, cinco nińos y adolescentes y una mujer embarazada. Todos eran bolivianos.

De acuerdo a Juan, es ahí donde empieza a generarse el colectivo denominado Simbiosis Cultural, precisamente después de ese incendio y después de la mediatización de conceptos como 'trabajo esclavo', 'migrantes ilegales', etc; con lo cual empiezan a trabajar y analizar,

[...] lo primero que nos salió fue contar, contar todas las situaciones que vivíamos los talleres textiles, romper un poco ese hito que estaba marcado

4 Disponible en: https://adiaria.com.uy/articulo/2017/3/jovenes-bolivianos-en-buenos-aires/. Acceso en: 27 sept. 2021. 
muy fuerte y en medio de ese contar que para nosotros fue muy importante, fue de la empezar a tomar la palabra, ahí empezamos a entender que en realidad la forma en que se había contado esta industria textil, de la forma en que se había mediatizado, estaba como muy fácil, muy sencilla y por lo tanto no visibilizaba un montón de cosas que hacen que ese sistema funcione. (JUAN, 2014-2017).

Este hecho es un gran avance, porque permite salir de la mirada centrada en el taller y sus formas de trabajo para comprender toda la cadena productiva en la cual se inserta y cómo es que recae todo el peso de la explotación en el trabajador textilero.

Nos juntamos en 2007 después del incendio del taller clandestino (ubicado en Luis Viale al 1200) ocurrido un año antes, donde seis personas murieron. Todos veníamos de trabajar en talleres textiles y no nos sentimos representados de la forma que se abordaba el tema de la precarización laboral. Por eso buscamos nuevas respuestas.

Cuando en 2015 se incendió otro taller clandestino, donde murieron dos niños, empezamos a contar las problemáticas que teníamos al trabajar en esa situación. La organización (Simbiosis Cultural) es nuestro faro para visibilizar la problemática tanto por lo coyuntural, como lo que nos pasa hace tiempo con la explotación laboral (Juan Vásquez).

El rubro textil fue y es un tema troncal en el colectivo, les atraviesa a todos, pero de distintas formas. Fue así que se empezó a tratarlo y ahí se hizo inevitable reconocer miles de parámetros con los cuales se tienen que enfrentar: la vivienda-taller, las articulaciones entre medios de producción y el folcklorismo, sus espacios de "contención", sus formas de ver la política, el adormecimiento sin fin de las radios, etc. En la parte final de su manifiesto expresan:

En ese recorrido nos potencian las incipientes rebeldías en el que nos vemos reflejados; las luchas por la diversidad sexual, las ganas de ocupar las calles al grito de justicia.

Pero el primer gran paso fue salir de la mirada y la postura victimista.

A partir de allí se tuvieron algunas cosas más claras.

Pasamos horas hablando por radio, cosiendo libritos, editando...

Eso somos, interrogantes, dudas, broncas, luchas, todas juntas y revueltas. Somos unxs jodidxs bolitas 5 .

5 Disponible en: https:/ladiaria.com.uy/articulo/2017/3/jovenes-bolivianos-en-buenos-aires/. Acceso en: 27 sept. 2021. 
El Colectivo Simbiosis Cultural es a nuestro juicio un ejemplo de nuevas formas organizativas de migrantes bolivianos que desde la auto-reflexión van construyendo nuevos espacios de resistencia y propuestas no sólo al habitar en un contexto diferente, sino sobre todo a repensarse desde el posicionamiento identitario laboral, social y político en el espacio que se habita. En todo caso, desde hace una década atrás en Argentina existe una construcción social y política de nuevos sujetos migrantes.

\section{La emergencia de la dimensión de género}

La migración de mujeres bolivianas hacia Argentina ha sido históricamente parte de un proyecto migratorio familiar, y si bien en los últimos años aumentó el número de mujeres que arribaron solas, todavía la mayor parte de este flujo se desplaza hacia este país en contextos familiares, de ahí la importancia de la familia para analizar el rol de la mujer boliviana en este proceso, y la persistencia de relaciones de género desiguales en ámbitos familiares y sociales.

Si bien los procesos migratorios permiten a las mujeres bolivianas opciones de generación de recursos económicos de manera autónoma reposicionándolas desde un rol más activo en el seno de sus familias, también parece ser que su inserción laboral tiende a ser provisional, lo cual incide en cierta medida en sus retornos a la comunidad.

En el relato de Delia Colque, migrante de la ciudad de El Alto y una de las fundadoras del Colectivo Simbiosis Cultural, se verbaliza y devela la dimensión de la violencia de género como elemento estructural de las migraciones de mujeres paceñas hoy en día:

[...] Vine a trabajar, pero también escapando de una situación bastante compleja que vivíamos en nuestro seno familiar, escapando de la violencia machista en la cual estábamos viviendo sobre todo por parte de mi papa hacia nuestra familia hacia mi mama ... pero sobre todo a nosotras dos hacia mi mama y yo que éramos las mujeres [...]

En la cuestión de cómo nosotros las mujeres migrantes nos vamos organizando, lo que vemos es que muchas mujeres migrantes nos organizamos y nucleamos en diferentes organizaciones sociales, políticas, feministas, de derechos humanos, pero como que todavía falta esto de la organización propia de mujeres migrantes ... pero lo importante es que de alguna manera nosotras nos vamos nucleando a los espacios que tenemos más cercanos y el empezar a pelear por nuestros derechos (DELIA, 2014-2017). 
El tema de la violencia de género como una constante invisibilizada en las dinámicas migratorias es otro eje central de la articulación de Simbiosis Cultural, sobre todo a partir de los casos emblemáticos de Marcelina Meneses ${ }^{6}$ en 2001 y Reina $\mathrm{Maraz}^{7}$ en 2010. En estos dos casos hallamos muy claramente esbozados las dimensiones de la tremenda violencia estructural que se ejerce a nivel del sistema sobre el cuerpo de las mujeres migrantes. Cada una de estas historias desde sus especificidades pone a luz cómo la mujer migrante está expuesta a una serie de vulneraciones y violencias en el ámbito familiar, así como en diversas esferas de lo sociales e institucional; pero a la vez cómo de estas situaciones extremas devienen capacidades solidarias, organizativas y reivindicativas fuertemente politizadas y movilizadoras.

Retomando las palabras y sentimientos de Delia Colque del Colectivo Simbiosis Cultural e integrante de 'Ni una migrante menos', en la Argentina está naturalizada la violencia hacia la mujer y también a los hijos, en los feminicidios que se generan en Argentina se encuentran también mujeres bolivianas asesinadas por sus parejas, la mayoría no se anima a hacer la denuncia. La policía, así como la justicia terminan recibiendo los casos y como hemos visto violentando aún más la condición de mujeres.

De esta manera y en los últimos tiempos de forma casi paralela las reivindicaciones laborales en torno a las condiciones en los talleres textiles y las reivindicaciones sobre violencia de género se fueron articulando en el accionar del Colectivo Simbiosis Cultural. Ambos aspectos se vieron fuertemente potenciados con la llegada al gobierno de Mauricio Macri el año 2015, cuya gestión se caracteriza entre otros elementos por el intento de criminalizar y sancionar

\footnotetext{
${ }^{6}$ El 10 de Enero del 2001, Marcelina Meneses y su hijo Joshua de diez meses viajaban en el tren rumbo al hospital y en el viaje fueron agredidos por su nacionalidad mediante insultos xenófobos y terminaron muertos al costado de las vías luego de ser arrojados del tren en movimiento. Las circunstancias de sus muertes tienen connotaciones racistas y xenófobas. El caso fue publicado mediáticamente a nivel nacional e internacional, para denunciar el caso se realizaron marchas durante un año todos los días 10 de cada mes. La causa, años después, se ha cerrado por falta de testigos y solo se presentó un único testigo, que fue descalificado por la fiscalía del departamento Judicial de Lomas de Zamora UFI No 1, provincia de Buenos Aires.

7 La historia de Reina Maraz, mujer migrante de una comunidad quechua en Chuquisaca (que al igual que muchas mujeres de comunidades rurales en Bolivia fue criada hablando sólo el idioma materno) casada y madre a los 17 años en un matrimonio violento. Llega obligada por el marido con sus dos niños el año 2009 a Argentina. Reina Maraz declaró que su marido solía golpearla estando borracho cuando vivían juntos en Bolivia. Una vez que estuvieron en la Argentina, los abusos continuaron donde la familia del marido apañó las acciones de éste además de quedarse con sus documentos. El 16 de noviembre de 2010, la mujer, embarazada de su tercer hijo, denunció en una comisaría de Florencio Varela la desaparición de su marido. A los cuatro días el cuerpo sin vida de Santos apareció y ella fue detenida y acusada del asesinato del marido. Pese a hablar solamente quechua y no comprender español, Reina Maraz fue retenida y sentenciada a cadena perpetua. Finalmente, el 30 de diciembre de 2016 fue absuelta por la Cámara de Casación Penal, que consideró que la mujer no había accedido a su legítima defensa. El caso de Reina Maraz es un caso emblemático de las formas en que la cultura dominante y el sistema judicial tratan a las mujeres, especialmente a las mujeres indígenas.
} 
a los migrantes mediante políticas públicas (DNU 70/2017). Por otro lado, también negando acceso a derechos a las mujeres en la temática del aborto y que desde las mujeres migrantes se vieron también interpeladas hasta constituir un bloque denominado "Ni una migrante menos" conformado por jóvenes mujeres migrantes, que deciden visibilizar las problemáticas ligadas a la migración y al ser mujer. Elementos que quedan claramente expresados en su declaración y en la opinión de sus integrantes, como Delia (2014-2017):

[...] fue a partir de la convocatoria que empezamos a tener para lo del DNU, aunque ya en otras ocasiones nos habíamos ido juntando con alguna de estas compañeras que son parte del bloque, sobre todo en los encuentros nacionales de mujeres que se hace todos los años allá, en Argentina; para eso, para las diferentes movidas de mujeres [...] nos íbamos articulando pero aún muy dispersas.

Yo creo que ahora, una vez que salió la Convocatoria para lo del paro migrante $[\ldots]$ nosotras tratamos de organizarnos con un mes y medio de anticipación. Es ahí donde nosotras tomamos como principales espacios de ir e intervenir; ahí nos vamos juntando con algunas compañeras más, como que recién estamos arrancando con la movida, luego tomamos la decisión de como mujeres migrantes ser parte de la organización 'Ni una Menos'.

En la declaración se posesionan como mujeres migrantes, provenientes de diferentes países de la región, que viven y sufren los efectos del patriarcado más allá de las fronteras nacionales. Por esa situación, afirman y asumen la lucha feminista que trasciende las fronteras. Denuncian en primera instancia al DNU por vulnerar sus derechos y puntualizan los efectos nocivos del capitalismo xenófobo, misógino y patriarcal que explota al ser humano y al trabajador en particular.

Como mujeres, migrantes y trabajadoras, denuncian una triple opresión que les pide ejercer con plenitud sus derechos y, entre éstos, a gozar de la igualdad jurídica tomando como referente el caso de Reina Maraz. Nuevamente vemos en esta referencia la influencia, al interior de los movimientos femeninos, de las migrantes bolivianas en el nacimiento y constitución del bloque "Ni una Migrante Menos". 


\section{El 'Bloque de Trabajadorxs Migrantes'}

En enero de año 2017, a inicios del gobierno de Mauricio Macri, se lanza el Decreto de Necesidad y Urgencia 70/2017 que fue presentado como una medida de seguridad y habilita la expulsión de personas migrantes, incluso por faltas migratorias y delitos leves. En ese sentido, asocia la migración con la delincuencia sobre un diagnóstico basado en datos parciales y descontextualizados. El cambio de paradigma apunta a la detención y la expulsión en remplazo de la regularización. Esta medida fue en gran medida el detonante para la constitución de una nueva organización migrante con características muy novedosas denominada "Bloque de Trabajadores Migrantes". El BTM se organiza a principios del año 2017 como una unión de diversos colectivos de jóvenes migrantes en torno a un hecho cohesionador: denunciar los peligros del DNU y concientizar sobre de los efectos en términos de xenofobia. De acuerdo a lo que se puede leer en la página digital del $\mathrm{BTM}^{8}$ se sostiene que:

Somos una organización conformada por distintos colectivos migrantes que nos organizamos para reivindicar nuestros derechos como sujetxs políticxs y culturales que hacemos parte activa de la sociedad argentina hoy y siempre!

\section{¡MIGRAR ES UN DERECHO!}

En marzo del 2017, nos agrupamos para hacer el primer Paro Migrante en Argentina. Posteriormente nos conformamos como Bloque de Trabajadoras y Trabajadores Migrantes (BTM), en respuesta a la embestida del gobierno macrista contra el derecho a migrar. Reivindicamos el ser parte del motor de la identidad cultural y política de todos los países. Lxs migrantes históricamente hemos representado una fuerza política y productiva fundamental y somos parte de la lucha con la cual se han logrado grandes conquistas en materia de derechos económicos, sociales, culturales, civiles y políticos.

El BTM pretende defender y luchar por los derechos que como migrantes y trabajadores/as hemos conquistado. Diferentes gobiernos de turno nos han utilizado como chivo expiatorio para ocultar sus responsabilidades en las sucesivas crisis económicas y políticas que desatan sobre los hombros del pueblo

8 Disponible en: https://www.facebook.com/bloquedetrabajadorxsmigrantes1/. Acceso en: 6 oct. 2021. 
trabajador. Creemos que los/las migrantes en Argentina debemos coordinar una resistencia conjunta frente a la ofensiva de los gobiernos.

De acuerdo a Natalia Gavazzo, el Bloque de Trabajadorxs Migrantes está conformada por:

Organizaciones lideradas por jóvenes migrantes o hijos de inmigrantes latinoamericanos buscaron generar un espacio de confluencia en torno a una propuesta novedosa: realizar el primer Paro Migrante. A partir de una conflictiva relación con las asociaciones de migrantes más tradicionales [...] se unieron para visibilizar los riesgos del DNU, y elaborar un discurso propio respecto de sus derechos a migrar y vivir dignamente en Argentina. Bajo las banderas "trabajadorxs migrantes presentes", "ni una migrante menos" o "derogación del decreto xenófobo", hasta consignas relativas a la dictadura y la democracia en los países de origen, y los derechos humanos, estos jóvenes bolivianos, colombianos, mexicanos, peruanos, paraguayos, brasileños, chilenos y salvadoreños, entre otros, marcharon en 2017 junto a diversos sectores sociales de la política argentina (GAVAZZO, 2018, p.136).

Más de 30 agrupaciones de inmigrantes, comunidades de varias naciones y trabajadores extranjeros que residen en Argentina convocaron y por primera vez, un Paro de Migrantes para denunciar al DNU, pero también visibilizar los aportes que realizan los migrantes en el país a nivel económico, social y cultural. El objetivo del Paro era pedir la derogación del Decreto 70/2017, que limita las garantías constitucionales de los residentes extranjeros. Las comunidades de Paraguay, Senegal, España, Brasil, Cuba, Colombia, Perú, Armenia, Uruguay y Bolivia prepararon durante dos meses el Paro, a partir de reuniones entre agrupaciones de extranjeros y trabajadores independientes, para hacer frente a la xenofobia que crecía en la Argentina. El país donde si bien la discriminación, precarización laboral y exclusión existieron desde hace años, pero que en el gobierno de Mauricio Macri se potenciaron ya sea a nivel discursivo como con medidas concretas y represión callejera (sobre todo hacia la comunidad senegalesa que se caracteriza por la venta callejera).

La fecha del primer Paro Migrante (30 de marzo) fue elegida en conmemoración del incendio del 30 de marzo de 2006, ocurrido en un taller clandestino de Caballito. En este hecho podemos notar la fuerte influencia de jóvenes bolivianos en los momentos fundacionales del Bloque. Juan Vásquez, en referencia al "Paro Migrante" señalaba que 
[...] con las organizaciones de distintos países planificamos que el 30 de marzo se realice un paro de migrantes con una concentración en el Congreso Nacional a partir de las 4 de la tarde, para que podamos visibilizar el contexto de crisis en el cual estamos viviendo. Uno de los principales temas que nos preocupa fue la inauguración de un centro de detención para migrantes y la precarización de nuestros derechos [...] Nosotros planteamos que los decretos que está llevando a cabo este Gobierno aceleran el proceso de deportación, implicando la precarización de los derechos de los inmigrantes. Este Paro nos posiciona de otra manera. Creemos que somos un gran aporte a la economía de este país (JUAN, 2014-2017).

En el año 2018, el Bloque de Trabajadores Migrantes convocó a un segundo Paro Migrante para el 4 de septiembre nuevamente en demanda de la anulación del DNU y al cese de persecución violencia contra migrantes, sobre todo a los migrantes senegaleses que en los últimos tiempos fueron los más perseguidos y reprimidos por la policía, en virtud a su actividad laboral en las vías públicas (venta ambulante) pero también por el color de su piel en clara muestra de prácticas institucionalizadas de racismo y discriminación.

Es importante señalar que desde la constitución del BTM su interrelación con diversos actores de la política local y nacional en varios ámbitos de la coyuntura argentina es muy estrecha. Es más aún en la situación de emergencia sanitaria, producto de la pandemia que a nivel internacional atravesamos, ya que desde la solidaridad se está actuando en campańas y acciones de apoyo a migrantes mas vulnerables.

\section{Los nuevos rostros}

Ya para finalizar y caracterizar más puntualmente a estos nuevos "sujetos políticos migrantes" que emergen en el presente siglo, debemos señalar los rasgos centrales del mismo. En primer lugar, destacar la ruptura que significa salir del gueto nacionalista. Es decir que el ámbito de acción e interrelacionamiento les ha generado una capacidad de articulación con otros colectivos, organizaciones, sindicatos o movimientos de migrantes, pero también y sobre todo de organizaciones locales. Esta capacidad de articulación queda expresada en la noción de autodenominarse: Bloque de Trabajadorxs Migrantes. Resulta un hito que el ethos organizativo ya no resida en la dimensión nacionalista de estos nuevos colectivos. Nos hallamos ante un viraje de lo nacional a lo laboral como nuevo eje articulador de las reivindicaciones. En otros términos, estamos ante el surgimiento de 
una "identidad trabajadora migrante" que se va forjando al calor de las protestas contra el endurecimiento de las políticas migratorias en la Argentina.

Es importante destacar que en muchos de estos espacios de articulación existe un rescate de la noción de 'comunidad' emanada del mundo andino, pero que presenta también sus propias particularidades de la dimensión transnacional y las articulaciones que ello supone, haciendo de estas comunidades espacios muy particulares. En segundo lugar, y mostrando un giro discursivo caracterizado tradicionalmente por la imagen victimista del migrante, hoy en día, hay una valoración del migrante como actor económico. Es un cambio discursivo fundamental en estas nuevas organizaciones, ya que frente a la victimización en la lucha contra la xenofobia o la discriminación se plantea visibilizar los aportes económico de la presencia migrante. En tal sentido, existe un reconocimiento en tanto sujeto de derechos, pero también como actor económico. Hoy en día tanto la horticultura como en la manufactura se hallan en manos bolivianas, si no hubiera horticultores bolivianos no se podría comer verdura barata en Argentina y lo mismo se podría afirmar de la ropa. Así de importante en términos económicos es la presencia de la mano de obra boliviana. En todo caso es claro que en Buenos Aires existe una nueva clase media boliviana ligada a estos dos rubros productivos que en cierta medida han posibilitado procesos de ascenso social de los migrantes bolivianos. Esta ruptura supone también desmitificar esa imagen que exclusivamente vincula al boliviano con el trabajador humilde racialmente muy identificable, sumiso, que no reclama y está siempre predispuesto para trabajar o, paralelamente con la fiesta y el baile folclórico que caracteriza esa ya tradicional presencia de los nacionales en Argentina. Por otro lado, directamente vinculados a los hechos analizados tanto de explotación laboral como de violencia de género fueron surgiendo una serie de organizaciones de diversas características y duración en el tiempo, pero que expresan nuevas formas de organización y actuación en lo político. Sin embargo, la creación desde el seno del Colectivo Simbiosis Cultural de la Cooperativa Juana Vilca (nombre en honor a la mujer embarazada muerta en el incendio de Luis Viale) es el mayor ejemplo de esta nueva situación. Vale decir, nuevas formas organizativas de jóvenes migrantes, pero que a la vez generan también organizaciones económico-productivas como búsqueda de respuestas a su situación de explotación laboral estructural a la cual están sometidos por las formas contemporáneas de organización productiva, en la cual la manufactura textil se halla inmersa. En todo caso, siendo los espacios productivos (talleres textiles) a su vez espacios organizativos y reivindicativos de orden político es claro que toda la reflexión sobre su situación, tenga también un correlato en la práctica productiva. Otra 
característica de estos espacios novedosos de organizaciones migrantes es que involucra mayormente a jóvenes (mujeres y hombres). Esta es un rasgo central de estos nuevos rostros de la bolivianeidad en Argentina. Son dinámicas y procesos que devienen de acciones y emprendimientos realizados básicamente por grupos de jóvenes y sus organizaciones que también representan innovaciones respecto a las clásicas organizaciones de residentes bolivianos. Se trata básicamente de colectivos de jóvenes cuyo funcionamiento y horizontalidad en su composición y accionar le confieren novedosas características. Es importante subrayar que el nivel de instrucción de estos jóvenes es mayor respecto a migrantes de anteriores décadas. Como mencionábamos al inicio de este último capítulo la temática de las segundas generaciones (hasta terceras), es decir, los hijos de migrantes y sus procesos y contextos de socialización/organización/reproducción y sobre todo politización, son elementos claves en estas reconfiguraciones que se vienen dando. Resulta central destacar que los espacios de información, relacionamiento y vinculación del conjunto de estos jóvenes ocurre básicamente a nivel de redes sociales e internet.

Pero quizá la mayor potencialidad y fuerza de estás nuevas emergencias sociales ligadas a la migración proviene de lo femenino como sustancia del "sujeto/actor migrante". La violencia de género en los espacios familiares como factor estructural de la migración de mujeres, la violencia de género en los espacios productivos de los talleres de costura que sumado a procesos de feminización de las migraciones, mayor segregación y explotación económica y laboral para las mismas, dio lugar a una emergencia significativa de 'lo femenino' como una de las principales potencias actuales del movimiento migrante en Buenos Aires, ya sea denunciando la reproducción de la violencia y las desigualdades de género en los espacios migratorios como también movilizándose y denunciando las políticas públicas atentatorias de los derechos de los y las migrantes.

\section{BIBLIOGRAFÍA}

BENENCIA, R. Migrantes bolivianos en la periferia de ciudades argentinas: Procesos y mecanismos tendientes a la conformación de territorios productivos y mercados de trabajo. In: NOVICK, S. (comp.). Las migraciones en América Latina: políticas, culturas y estrategias. Buenos Aires: Catálogos, 2008. p. 13-30.

BENENCIA, R. Información y redes sociales en la conformación de mercados de trabajo: La migración en la horticultura periurbana de la Argentina. In: CONGRESO 
LATINOAMERICANO DE SOCIOLOGÍA DEL TRABAJO, 5., Montevideo, Uruguay. Anales [...], Montevideo, Uruguay, abr. 2007. p. 24-31.

BENENCIA, R. Migración limítrofe y mercado de trabajo rural en la Argentina: estrategias de familias bolivianas en la conformación de comunidades transnacionales. Revista Latinoamericana de Estudios del Trabajo, Buenos Aires, año 10, n.17, 2005.

BENENCIA, R. Familias bolivianas en la producción hortícola de la Provincia de Buenos Aires: Proceso de diseminación en un territorio transnacional. In: HINOJOSA, A. (comp.). Migraciones transnacionales: Visiones de Norte y Sudamérica. La Paz: Plural/CEPLAG-UMSS, 2004. p. 201-230.

BENENCIA, R. De peones a patrones quinteros. Movilidad social de familias bolivianas en la periferia bonaerense. Estudios Migratorios Latinoamericanos, Buenos Aires, año 12, n. 35, 1997.

BENENCIA, R.; KARASIK, G. Inmigración limítrofe: Los bolivianos en Buenos Aires. Buenos Aires: Editorial CEAL, 1995.

CAGGIANO, S. Cuerpos y escenarios de la migración: Clase, raza y género en las imágenes visuales de bolivianos/as en la prensa. In: COLOQUIO INTERNACIONAL MIGRANTES REGIONALES EN LAS AMÉRICAS, 2008, Buenos Aires. Anales [...], Buenos Aires: Instituto de Desarrollo Económico y Social, 2008.

CAGGIANO, S. Lo nacional y lo cultural: Centro de estudiantes y residentes bolivianos: representación, identidad y hegemonía. In: DOMENECH, E. (comp.). Migraciones contemporáneas y diversidad cultural en la Argentina. Córdoba: Centro de Estudios Avanzados, Universidad Nacional de Córdoba, 2005a. p.155-186.

CAGGIANO, S. Lo que no entra en el crisol: Inmigración boliviana, comunicación intercultural y procesos identitarios. Buenos Aires: Prometeo Libros, 2005b.

CAGGIANO, S. ¿Bolivianos? En dos ciudades ¿argentinas?: Identidades sociales en procesos migratorios contemporáneos". In: CLACSO. Informe Final del concurso: culturas e identidades en América Latina y El Caribe. Buenos Aires: Programa Regional de Becas, 2001.

DELIA. Colectivo Simbiosis Cultural. Entrevistador: Alfonso Hinojosa. La Paz, Buenos Aires, 2014-2017. Entrevistas realizadas en varios momentos.

DOMENECH, E. (comp.). Migraciones contemporáneas y diversidad cultural en la Argentina. Buenos Aires: Universidad Nacional de Córdoba, Centro de Estudios Avanzados, 2005. 
DOMENECH, E.; MAGLIANO, M. J. Género, política y migración en la agenda global. Transformaciones recientes en la región Sudamericana. Migración y Desarrollo, Zacatecas, n. 12, 2009.

GAVAZZO N. Jóvenes migrantes e hijos de inmigrantes latinoamericanos en Buenos Aires: una generación en movimiento, Confluenze, Bologna, v.10, n.1, p. 131-165, 2018.

GAVAZZO, N. El valor de la cultura en la acción política: Fenómenos migratorios y producción artística. Revista Encrucijadas, Buenos Aires, n.57, 2016.

GAVAZZO, N. Hijos de bolivianos y paraguayos en el área metropolitana de Buenos Aires: Identificaciones y participación entre la discriminación y el reconocimiento. 2014. 290f. Tesis (Doctorado en Antropología) - Facultad de Filosofía y Letras, Universidad de Buenos Aires, Buenos Aires, 2014.

GAVAZZO, N. Para todos los hombres del mundo: diversidad cultural y nación en algunos discursos públicos sobre la inmigración en Argentina. In: VIANA GARCES, A. (coord.). Repensar la Pluralidad. Madrid: Fundación Universidad Carlos II y Editorial Tirant Loblanch, 2009.

GIORGIS, M. La virgen prestamista: La fiesta de Urkupińa en el boliviano gran Córdoba. In: HINOJOSA, A. (comp.). Migraciones transnacionales: visiones de Norte y Sudamérica. La Paz: Plural/CEPLAG-UMSS, 2004. p. 141-166.

GREGORIA. Colectivo Bolivia Marka. Entrevistador: Alfonso Hinojosa. Córdoba, 30 de marzo 2006.

GRIMSON, A. Relatos de la diferencia y la igualdad: Los bolivianos en Buenos Aires. Buenos Aires: Eudeba, 1999.

GRIMSON, A.; PAZ SOLDÁN, E. Migrantes bolivianos en la Argentina y los Estados Unidos. Cuadernos de futuro, La Paz, n.7, 2000.

INSTITUTO NACIONAL DE ESTADÍSTICA [INE]. Censo Nacional de Población y Vivienda de 2012. Base de datos. Disponible en: https://nube.ine.gob. bo/index.php/s/bROAGTD7pFvBP1f/download. Acceso en: 5 oct. 2021.

JUAN. Colectivo Simbiosis Cultural. Entrevistador: Alfonso Hinojosa. La Paz, Buenos Aires, 2014-2017. Entrevistas realizadas en varios momentos.

ORLANDO. Colectivo Bolivia Marka. Entrevistador: Alfonso Hinojosa. Córdoba, 24 de marzo 2006. 
Jóvenes migrantes y política: los nuevos rostros de la bolivianeidad en Argentina

SASSONE, S. et al. Migrantes bolivianos y horticultura en el Valle Inferior del río Chubut. HINOJOSA, A. (comp.). Migraciones transnacionales: visiones de Norte y Sudamérica. La Paz: Plural/CEPLAG-UMSS, 2004. p. 231-269.

Recebido em: 06 de setembro de 2020

Aprovado em: 23 de novembro de 2020 